REGARDS

SUR L'ECONOMIE ALLEMAND

BULLETIN ECONOMIQUE DU CIRAC
Regards sur l'économie allemande

Bulletin économique du CIRAC

$101 \mid 2011$

Varia

\title{
Sortie du Nucléaire - quelques pistes de réflexion
}

Isabelle Bourgeois

\section{CpenEdition}

\section{Journals}

Édition électronique

URL : http://journals.openedition.org/rea/4296

DOI : 10.4000/rea.4296

ISBN : 978-2-8218-0954-3

ISSN : 1965-0787

Éditeur

CIRAC

Édition imprimée

Date de publication : 10 juin 2011

Pagination : 33-37

ISSN : 1156-8992

Référence électronique

Isabelle Bourgeois, "Sortie du Nucléaire - quelques pistes de réflexion 》, Regards sur l'économie allemande [En ligne], 101 | juin 2011, mis en ligne le 19 septembre 2011, consulté le 15 septembre 2020. URL : http://journals.openedition.org/rea/4296 


\section{Actualité économique}

\section{Sortie du Nucléaire - quelques pistes de réflexion}

Le 6 juin, le gouvernement fédéral votait, en conseil des ministres, les projets de loi accompagnant sa nouvelle stratégie en matière de mix énergétique : "La voie vers l'énergie du futur " (Weg zur Energie der Zukunft). Celle-ci prévoit, d'ici 2020, d'amener à $35 \%$ la part des sources d'énergie renouvelables dans la production d'électricité (actuellement de $17 \%$; objectif $2050: 80 \%$ ), de réduire de $10 \%$ la consommation d'électricité, de réduire de $40 \%$ par rapport au niveau de 1990 les émissions de $\mathrm{CO}_{2}$ (puis de $80 \%$ à $95 \%$ d'ici 2050) et de renoncer définitivement au nucléaire en 2022.

Le 3 juin, Bund et Länder étaient parvenus à un accord sur les principales modalités de sortie du nucléaire - essentiellement sur un échelonnement entre 2015 et 2022 de l'arrêt des 9 centrales encore en activité en Allemagne. Le 30 mai, le gouvernement fédéral avait annoncé sa décision de mener une nouvelle politique énergétique basée sur l'abandon irrémédiable du nucléaire en 2022 ; le projet prévoyait deux vagues d'arrêt pour ces 9 centrales, l'une en 2021, l'autre un an plus tard. Le 15 mars (une semaine avant les élections dans le Bade-Wurtemberg), le gouvernement fédéral avait adopté un " moratoire nucléaire » (Atom-Moratorium) ordonnant, pour des raisons de sécurité, l'arrêt immédiat des huit centrales les plus anciennes (sur les 17 au total) jusqu'au 15 juin. Le 30 mai, il a été changé de cap et décidé qu'elles ne seront jamais remises en service ; décision confirmée le 6 juin.

Quelques mois plus tôt seulement, en septembre 2010, le gouvernement fédéral avait à l'inverse conclu un accord avec les énergéticiens sur le prolongement (entre 8 et 14 ans) de l'exploitation de 16 centrales et avait fixé ce faisant la date d'abandon du nucléaire à 2036. Cet accord amendait la Loi sur l'atome (Atomgesetz) en vigueur depuis 2002 et qui avait été votée à la suite de l'accord sur la sortie du nucléaire conclu en juin 2000 sous la coalition SPD/Verts (voir REA 41/1999). Cette loi ne prévoyait pas de date butoir, mais l'échelonnement de l'extinction des centrales s'étendait jusqu'à l'horizon 2036.

L'Allemagne sera donc dénucléarisée d'ici dix ans. L'avarie du réacteur de Fukushima a profondément marqué les esprits. L'opinion publique allemande, qui avait cédé à la panique après l'accident de Tchernobyl, a vu raviver ses peurs irrationnelles (voir l'interview d'actualité consacrée à ce thème sur www.cirac.u-cergy.fr). Même la chancelière Merkel, physicienne de formation, a été amenée à réviser sa réflexion sur le risque et la part de risque acceptable, ainsi qu'elle l'explique dans un long entretien accordé à l'hebdomadaire Die Zeit (12-05). "En tirer les nécessaires conséquences peut, pour la première fois, donner lieu à un vaste consensus sur cette question, et souder la société ". La chancelière aurait-elle cédé au populisme? A-t-elle cédé à l'électoralisme pour sauver son parti face à la montée en puissance des Verts dans les élections régionales et dans l'opinion? Ou y a-t-il d'autres raisons poussant l'Allemagne à accélérer aujourd'hui sa sortie du nucléaire? Voici quelques pistes de réflexion stratégique.

\section{Adhésion des milieux économiques}

Les premières réactions des milieux économiques à l'annonce, le 30 mai, de la date rapprochée de sortie du nucléaire sont virulentes. Dieter Zetsche, président du directoire du groupe Daimler, s'insurge contre "l'absence de cap politique lisible » et un choix " présentant certains risques pour un site industriel comme l'Allemagne " (BILD, 30-05). "Nous nous livrons à des expériences sur la compétitivité de notre économie - avec une issue incertaine », fulmine le patron de l'électricien RWE, Jürgen Grossmann (BILD, 31-05). Quant à Hans-Peter Keitel, président de la fédération allemande de l'industrie (BDI), il s'inquiète de la sortie irréversible du nucléaire, soulignant le risque d'une hausse des prix de l'électricité et de variations préoccupantes pour la sécurité de l'approvisionnement (communiqué du 30-05; www.bdi-online.de).

Curieusement, même plusieurs jours après l'annonce, ces réactions se limitent à quelques rares petites phrases relayées en boucle par les médias. On chercherait en vain le 'grand classique' allemand de la mise en garde du monde économique vis-à-vis de choix politiques considérés comme dommageables à la compétitivité : un appel conjoint des quatre grandes fédérations BDI, BDA (le 'Medef' allemand), DIHK (les CCI) et $\mathrm{ZDH}$ (l'artisanat). Les quelques critiques et protestations des patrons et présidents de fédérations restent en surface et, surtout, convenues : les règles d'une société aussi médiatisée que l'Allemagne exigent d'eux qu'ils alimentent le 'buzz'.

\section{Analyse}

30 mai : annonce de la fin du nucléaire en 2022

Septembre 2010 : prolongement du nucléaire jusqu'en 2036

Comment expliquer ce revirement?

Quelques phrases assassines dans les médias...

... mais pas de contre-offensive 
Colère des grands électriciens

Attitude constructive de l'industrie

A l'avenir, contrer les effets pervers de la Loi EEG

Addiction aux subventions?
Mais dans ce concert, on distingue deux catégories de voix. D'abord celle des grands électriciens (E.ON, RWE, EnBW et Vattenfall), les premiers concernés. Le brusque changement de cap de la coalition fédérale réduit à néant leurs stratégies et prévisions. A leurs yeux, il s'assimile à une rupture unilatérale de contrat - plus précisément la fermeture brutale et sine die de leurs centrales les plus anciennes, qui entraîne de lourdes pertes d'exploitation et de valorisation (22 milliards $€$ selon le quotidien Handelsblatt du 3 juin qui cite une étude réalisée par la banque LBBW). II les contraint par ailleurs à accroître leur facture de certificats d'émissions de $\mathrm{CO}_{2}$, puisque dans leur parc, la part de la production d'électricité 'polluante' s'accroît de ce fait. E.ON a donc signalé, RWE et Vattenfall ont annoncé, qu'ils allaient engager des procédures en dommages et intérêts. Leurs réactions sont de nature purement juridique et commerciale.

Différentes sont les voix (et motivations) des industriels. Qu'un fleuron de l'industrie automobile comme Daimler redoute une hausse du prix de l'électricité peut se comprendre à première vue, mais l'industrie forte consommatrice bénéficie d'ores et déjà d'une tarification préférentielle. La prise de position d'un D. Zetsche s'explique bien plus par les lois inhérentes au jeu médiatique : les journalistes vont recueillir systématiquement la 'petite phrase' de l'expert ou de la personnalité en vue ; or qui de plus 'représentatif' en l'occurrence que le patron de 'Mercedes'? En réalité, les industriels allemands adhèrent au nouveau cap poursuivi par le gouvernement fédéral : "L'industrie partage foncièrement la volonté politique d'une révolution énergétique », déclare ainsi Christopher W. Grünewald, président de la commission Politique énergétique et climatique de la fédération BDI (communiqué du 29-05). S'ils s'insurgent publiquement contre le fait de ne pas avoir été associés au processus de prise de décision qui a débouché sur l'accord du 30 mai mais il est permis d'en douter, tant les échanges entre industrie et pouvoirs publics sont institutionnalisés en Allemagne -, les industriels s'en accommodent par ailleurs. Ils savent que les décisions du conseil des ministres ne sont encore que des projets, qu'elles doivent être soumises à la procédure de débat parlementaire et que, alors, comme toujours dans le jeu institutionnel allemand, leurs réflexions seront prises en considération. Ecoutons les propos pragmatiques tenus par Hans-Peter Keitel, président du $\mathrm{BDI}$, dans une interview accordée à la radio Deutschlandfunk le 6 juin : " il faut distinguer la sortie du nucléaire qui est semble-t-il un consensus national, et que donc nous soutenons... et l'entrée dans une nouvelle situation énergétique ». Cette dernière est un processus " que nous devons gérer de manière raisonnée, sans précipitation... et sans mettre en danger notre prospérité ». Sans l'industrie, pas de 'révolution' énergétique...

\section{Une nouvelle politique industrielle?}

Cette attitude constructive s'attire néanmoins quelque suspicion, pas seulement dans la presse. Car les grands défis que subsument les formules rituelles de « développement durable " et de "lutte contre le changement climatique " sont générateurs de confortables subventions publiques. La loi sur les énergies renouvelables (Erneuerbare-Energien-Gesetz, EEG) du 29-03-2000, par exemple, a littéralement dopé le photovoltaïque qui ne représente pourtant en 2011 que $3 \%$ de la consommation totale d'électricité, mais $20 \%$ de la production soutenue par la Loi EEG. En dix ans, les installations photovoltaïques ont bénéficié au total de 85,4 milliards $€$ de subventions, s'insurge, dans une lettre adressée le 2 mai au ministre fédéral de l'Economie, le Conseil économique (Wissenschaftlicher Beirat) placé auprès de ce dernier dans le contexte de la révision de la Loi EEG qu'implique la nouvelle politique énergétique allemande. Dans le communiqué de presse du $1^{\text {er }}$ juin qui fait suite à cette lettre, le Conseil insiste : la réforme de la loi doit être l'occasion d'en corriger les effets pervers. "Le soutien de technologies particulières tel que le prévoit actuellement la Loi EEG mène à des distorsions en ce qui concerne les choix de l'industrie en matière de recherche et de production. Ce n'est pas la concurrence des inventeurs, mais la décision du législateur qui décide quel procédé alternatif de production d'énergie doit être prioritaire. Or cela est hautement risqué ». Cette loi s'est donc révélée contre-productive en termes d'innovation, puisqu'elle étouffe dans l'œuf la créativité et inhibe l'émergence d'approches et solutions nouvelles.

Par-delà la question du subventionnement ciblé de certaines sources d'énergie renouvelables plutôt que d'autres, une partie de la presse plutôt proche des libéraux et des chrétiens-démocrates regrette que la pratique des subventions à l'économie semble avoir le vent en poupe en Allemagne depuis la récession et les colossales mesures de soutien à la conjoncture adoptées dans ce cadre par le gouvernement fédéral. Et elle cite volontiers les calculs de l'institut IfW de Kiel qui consacre régulièrement des études à la question. Selon ces calculs, le volume total des subventions à l'économie est passé de 104,9 milliards $€$ en 2008 à 113,9 milliards $€$ en 2009 , avant de baisser légèrement pour s'établir à 111,9 milliards $€$ en 2010 , soit une part de $4,48 \%$ du PIB. 
Les éditorialistes défendant l'orthodoxie ordo-libérale constatent qu'aujourd'hui, alors que le moteur conjoncturel allemand tourne à plein régime, la légitimation de cette pratique a subi une inflexion : tandis qu'au plus fort de la crise financière mondiale, il s'agissait de préserver l'économie réelle, aujourd'hui, l'objectif est de soutenir la compétitivité du site Allemagne face à la montée en puissance des économies émergentes, surtout la Chine. La chancelière Merkel ne déclarait-elle pas, lorsqu'elle présentait à la presse la décision du conseil des ministres du 30 mai : "En se repositionnant sur les énergies renouvelables, l'Allemagne peut jouer le rôle de pionnier à l'international » ? Pour sa part, Klaus Mittelbach, président du directoire de la fédération des industries électriques et électrotechniques ZVEI - l'une des branches phares de l'industrie allemande et de sa compétitivité -, semble donner raison à ces analyses: "Notre monde n'est plus le même que voici vingt ans: le changement climatique, l'augmentation de la population mondiale et la concurrence dans une économie globalisée rendent nécessaire une aide financière au démarrage de la part de l'Etat », expliquait-il (F.A.Z., 30-05).

Beaucoup d'éléments portent à croire que l'habillage écologique d'une politique énergétique " sur la voie de l'avenir » se prête idéalement à justifier un revirement dans la doxa de la politique économique allemande et que l'Allemagne, face à l'hystérie collective née du risque nucléaire actuel dans un pays hautement industrialisé comme le Japon, est tentée de mener aujourd'hui une politique industrielle dirigiste et planifiée qui ne dit pas son nom. Face à cette nouvelle offre, la demande en matière de subventions ne semble pas en reste. Alors que jusqu'ici, ce sont surtout les grands groupes qui en bénéficiaient - le dernier exemple en date est celui des aides à l'industrie automobile pour développer les véhicules électriques -, certains professionnels se spécialisent dans la 'chasse aux subventions' à destination du Mittelstand (voir par exemple le site d'un cabinet de conseil comme www.brose-partner.de). Ces tendances de fond qui s'esquissent bien au-delà du secteur énergétique amènent un éditorialiste à s'insurger : "Dans l'Allemagne réunifiée, il n'y a jamais eu autant de RDA qu'aujourd'hui » (Welt Online, 28-05). Et un autre décèle " un adieu tacite à la libéralisation du marché de l'énergie décrétée voici 12 ans" qui « se solde par une renationalisation du marché de l'énergie et mène tout droit à une économie planifiée » dans ce secteur (F.A.Z., 06-06).

Leur crainte n'est pas totalement infondée. Dans son rapport remis au gouvernement fédéral le 30 mai, la Commission d'éthique (Ethik-Kommission) instituée ad hoc pour faire des propositions sur la sortie du nucléaire et un nouveau mix énergétique écrivait : "Les infrastructures seront la pièce maîtresse d'une économie high-tech ». Et elle ajoutait : " II ne faut plus voir les réseaux seulement sous l'angle de la libéralisation du marché..., mais les considérer dans leur fonction au service de l'intérêt général ". II est vrai que le segment du transport de l'électricité relève largement du privé, mais qu'il est encore peu optimal. Or, constitutionnellement les infrastructures relèvent du pouvoir régalien de l'Etat. La Commission n'appelle, au fond, qu'à une meilleure prise en considération de leur rôle stratégique pour l'alimentation en électricité.

\section{La question des infrastructures au cœur du nouveau mix énergétique}

La critique la plus féroce à l'encontre du projet de sortie du nucléaire émane de l'autorité de régulation des industries de réseaux, la Bundesnetzagentur. Lors de la conférence de presse du 27 mai au cours de laquelle elle présentait les conclusions de son rapport sur les conséquences de la fermeture immédiate des 8 centrales sur la sécurité de l'approvisionnement en électricité, l'autorité pointe du doigt le caractère anti-concurrentiel des mesures que sont contraints d'adopter les acteurs de la branche pour sécuriser production, transport et coûts. De ce fait, estime-t-elle, "l'objectif visé de la constitution d'un marché aux structures obéissant aux lois de la concurrence se trouve remplacé par une approche de planification et de pilotage plus ou moins centralisé ". Or, poursuit-elle, " cela est problématique pour le marché de l'énergie, inefficient pour l'économie et dommageable pour l'environnement ». Cependant, ces effets " actuellement incontournables peuvent être acceptés durant une période transitoire ". Mais l'autorité, qui coopère avec ses homologues des autres Etats de l'UE, rappelle aussi qu'en la matière, la coordination européenne reste indispensable. Et elle précise : "Rien ne justifie non plus de faire fi de [notre] mise en demeure de ne pas arrêter d'autres centrales pour des motifs purement politiques ou, plus précisément, de ne procéder à de telles mesures qu'après une étroite et minutieuse coordination avec les exploitants des réseaux de transports d'électricité et la Bundesnetzagentur ». Le seul "moratoire » génère en effet d'importants risques de black-out au plus tard dès cet automne.

Le gouvernement fédéral a tenu compte du rapport que lui avait remis la Bundesnetzagentur le 11 avril, suivi d'une actualisation le 26 mai. Informé des risques que les déséquilibres ou variations au niveau de la production et de la demande font peser sur la sé-
Etatisation rampante du marché de l'énergie?

Risques de black-out

Prise de conscience 
Pénurie de lignes à haute tension

Vers une nouvelle architecture de réseaux

2 pierres d'achoppement pour le marché européen

Cavalier seu ou éclaireur? curité de l'acheminement, le gouvernement semble plutôt avoir pris ce risque délibérément. Certes, une récente étude de I'Institut DIW conclut à l'absence d'un tel risque (DIW Wochenbericht, 20/2011), mais elle se concentre sur le seul aspect de la production d'électricité. Le gouvernement semble plutôt vouloir forcer la prise de conscience de l'importance stratégique des réseaux - à la fois en Allemagne et dans l'UE.

Outre-Rhin, le réseau de transport est largement sous-dimensionné et, encore plus, inadapté. Pour simplifier : la production éolienne se concentre dans le nord, alors que la demande se concentre au sud; et selon l'Agence allemande de l'Energie (DENA), il manque au moins $3600 \mathrm{~km}$ de lignes à haute tension à l'horizon 2020. Ce problème n'est pas nouveau (voir REA 72/05). Mais les électriciens rechignent devant le volume des investissements à réaliser, et les administrations publiques ne se montrent guère empressées à réduire la durée de délivrance des autorisations (elle est en moyenne de dix ans); quant aux riverains, frappés du syndrome du "nimby " (" not in my backyard »), ils s'opposent farouchement à l'érection de pylônes... Placer la branche devant le fait accompli pourrait dès lors accélérer la mise à niveau des infrastructures.

Dans le mix énergétique de l'avenir, une part centrale reviendra à l'éolien, notamment offshore ( 5 milliards $€$ d'aides sont prévues) - raison de plus pour renforcer les grandes liaisons nord-sud. Le gouvernement fédéral vise donc à modifier la répartition des compétences entre Bund et Länder en matière d'infrastructures pour mieux les planifier à l'échelon fédéral. Mais, bien que cela ne soit qu'à peine thématisé dans l'espace public, l'approche allemande va beaucoup plus loin. Dans son podcast vidéo du 4 juin, la chancelière explique en effet que le nouveau mix énergétique appelle " une architecture totalement nouvelle » du réseau électrique. Fermer d'une part les grandes centrales de production, en construire d'autre part une multitude de nouvelles, plus petites, à proximité de la demande des consommateurs (ménages ou industriels), et enfin stocker la surproduction d'électricité des éoliennes en fonction de la météo pour éviter une surcharge du réseau tout en répondant le cas échéant à une demande accrue à certains moments de l'année, tout cela est impossible avec l'architecture actuelle. Car cela exige une multitude de micro-réseaux répartis sur l'ensemble du territoire et reliés entre eux, de multiples infrastructures de stockage, ainsi qu'une gestion 'intelligente' de la production et des réseaux. Autrement dit, l'Allemagne semble miser sur le renforcement d'une structure réticulaire qui existe déjà partiellement sur son territoire et prend son essor dans le cadre notamment de l'essor de la filière hydrogène (voir REA 66/04), ainsi que sur le développement en force des technologies de smart grid. Car en l'état actuel, seules les technologies liées à l'hydrogène semblent permettre de gérer à la fois les variations de la production grâce au stockage (pile à combustible), d'optimiser l'apport des diverses sources renouvelables (biomasse, solaire, etc.) à la production totale et d'assurer la sécurité de l'approvisionnement. Les salons professionnels comme ce $f$-cell 2011 qui se tiendra à Stuttgart les 26-27 septembre apporteront de plus amples informations.

\section{Faire bouger les lignes pour accélérer l'intégration du marché européen de l'énergie}

C'est principalement sur la question des infrastructures qu'achoppe la constitution d'un " marché intérieur de l'énergie intégré, interconnecté et pleinement opérationnel » (Conclusions du Conseil européen, 04-02). Des «efforts considérables... sont essentiels si l'on veut que la solidarité entre les Etats membres devienne effective, que d'autres voies d'acheminement ou de transit et d'autres sources d'énergie deviennent une réalité concrète et que des sources d'énergie renouvelables se développent ". La politique énergétique européenne achoppe également sur la promotion des renouvelables et des technologies "décarbonnées". Le choix du mix énergétique n'est en effet pas une compétence communautaire, et les Etats membres peinent à définir une ligne commune (voir REA 82/07 et 90/09). De ce fait, le Commissaire à l'Energie, Günther Oettinger, cherche à définir une politique communautaire en la matière. Le projet s'attire l'opposition de l'Allemagne pour une raison fondamentale : cette communautarisation est contraire au principe de subsidiarité. C'est assurément aussi l'imminence d'une telle offensive de 'Bruxelles' qui aura incité le gouvernement fédéral à prendre les devants...

Le 'cavalier seul' de la République fédérale répond, contrairement aux apparences, au motif de l'approfondissement du marché européen. Du moins l'Allemagne cherche-t-elle à provoquer un sursaut sur un dossier d'importance stratégique pour la compétitivité industrielle de l'UE et de ses Etats membres: la question des interconnexions électriques au sein de l'UE. Elle use de sa prérogative nationale en matière de choix énergétiques pour faire progresser ce dossier communautaire. Tout en forçant le débat européen sur les renouvelables, dont l'hydrogène - une technologie d'importance stratégique pour la politique d'innovation et pour la compétitivité de l'UE face à la concurrence asiatique et qui était à l'honneur cette année à la Foire de Hanovre. L'Allemagne force aussi 
les industriels allemands à mener une politique plus européenne en matière de renouvelables - donc à investir plus à la fois dans la production (26,6 milliards $€$ d'investissements en 2010 ; DIW Wochenbericht, 20/2011) et dans la recherche sur les technologies 'décarbonnées - en priorité cette filière hydrogène dont l'UE a fait une de ses priorités.

\section{Une dénucléarisation consensuelle}

La sortie du nucléaire ayant été négociée avec les partis composant le gouvernement de coalition (CDU, CSU et FDP), très peu de voix s'élèvent contre dans ce camp. Arnold Vaatz, vice-président de la commission CDU/CSU au Bundestag, est l'un des rares à avoir ouvertement critiqué la sortie du nucléaire, la considérant comme " une catastrophe irréparable » pour l'Allemagne (Mitteldeutsche Zeitung, 30-05). Quant au FDP, il pointe timidement du doigt certaines tendances dirigistes...

L'opposition s'insurge. C'est pourtant elle qui avait posé les jalons de la sortie du nucléaire à partir de 1999. La décision du gouvernement Merkel se contente d'accélérer la fin du nucléaire programmée à l'horizon 2036 sous le chancelier Schröder. Les critiques portent dès lors dans un premier temps sur le détail des dispositions décidées le 30 mai. Kurt Beck (SPD), ministre-président de Rhénanie-Palatinat, s'exprimant au nom des Länder gouvernés par le SPD seul ou en coalition et qui sont aujourd'hui majoritaires au Bundesrat, se montrait réservé quant au vote de la chambre des Länder : « II est trop tôt pour dire que ce sera non. Mais je suis loin d'être sûr que nous porterons " le projet (Stuttgarter Zeitung, 31-05). Menace rituelle dans le cadre de négociations outre-Rhin. Claudia Roth, présidente des Verts, déclarait aux quotidiens du groupe WAZ (Essen, 3105) : "Nous attendons que soit présenté un programme réaliste pour la mise en arrêt de chacun des réacteurs ». Quant au ministre de l'Ecologie du nouveau gouvernement SPD/Verts du Bade-Wurtemberg, il affirmait : "Le risque est grand de voir resurgir un débat sur le prolongement de la durée d'exploitation dès 2019, quand se profilera l'arrêt simultané des 9 centrales alors encore en activité " (FAZ, 01-06).

Ces réserves n'ont plus lieu d'être depuis le 3 juin : les deux dates butoir (2020 et 2022) ont cédé la place à un échelonnement graduel dans le sens des Verts et du SPD. Pour éviter une panne de réseau, une ou plusieurs centrales resteront en stand-by ; mais au lieu d'une centrale nucléaire comme prévu initialement, il s'agira d'une ou plusieurs centrale(s) au gaz et au charbon. "Personne en Allemagne ne souhaite un black-out ", résumait la chancelière (F.A.Z., 03-06). Le compromis conclu ce jour-là entre le gouvernement fédéral et les Länder a débouché sur un classique compromis à l'allemande, préservant les intérêts du niveau fédéral et des Länder, du gouvernement et de l'opposition. II ne reste plus aux Verts qu'à semer le doute sur la faisabilité de cette 'révolution'.

La voie est libre maintenant pour la procédure parlementaire. Huit lois au total devront être amendées, autant de projets de loi adoptés le 6 juin. Ainsi, par exemple, le calendrier d'arrêt des centrales sera fixé par la Loi sur l'atome, la Loi sur l'économie de l'énergie (Energiewirtschaftsgesetz) étendra les prérogatives de la Bundesnetzagentur, notamment en matière d'investissement dans les infrastructures, la Loi $E E G$ sera modernisée... Un seul point important reste litigieux : la question de la répartition des compétences entre Bund et Länder en matière de planification et de construction des infrastructures. Ces amendements doivent être adoptés dans un laps de temps record: les débats au Bundestag doivent débuter le 17 juin et être clos le 30 juin ; le 8 juillet, les textes doivent être soumis au Bundesrat. Le dossier devrait être clos durant l'été.

\section{Faire bouger les lignes sur la carte politique}

La chancelière a fauché l'herbe sous les pieds des Verts qui avaient construit leur identité sur l'abandon du nucléaire depuis la genèse du mouvement écologique à partir du milieu des années 1970. C'était là indéniablement l'intention de la chancelière face à une impasse politique. Le FDP, partenaire de l'Union au sein de la coalition fédérale, est en proie à une profonde crise d'identité. Les Verts montaient en puissance, se positionnant de plus en plus au centre et attirant une partie croissante de l'électorat de la CDU/CSU (et du SPD). Maintenant que leur revendication mère est en passe de devenir réalité, sur quelle base vont-ils à l'avenir reconstruire leur identité ?

La chancelière a saisi l'opportunité que représentait l'hystérie anti-nucléaire de l'opinion allemande après 'Fukushima' pour rassembler et créer un consensus national sur ce qui était devenu une question de société. L'instauration d'une Commission d'éthique composée de représentants de la société civile en est le reflet. Dans son rapport, remis au gouvernement fédéral le 30 mai, elle souligne "que la révolution énergétique ne peut réussir que sur la base d'un effort conjoint à tous les niveaux du politique, de l'économique et de la société ". L'abandon du nucléaire, thème fédérateur s'il en est, ne pourrait-il pas dès lors préfigurer le choix d'une nouvelle Grande coalition ? (IB, 06-06-2011)
Les critiques de l'opposition sur les modalités...

... ont été intégrées dans l'accord du 3 juin

Vote parlementaire achevé cet été ?

Les Verts n'ont plus d'identité 